\title{
Cerebellar Pilocytic Astrocytoma
}

National Cancer Institute

\section{Source}

National Cancer Institute. Cerebellar Pilocytic Astrocytoma. NCI Thesaurus. Code C6809.

A WHO Grade 1 astrocytoma which arises in the cerebellum. The tumor is composed of spindle shaped cells with numerous collections of reddish astrocytic fibers called Rosenthal fibers. Over $80 \%$ or the cerebellar astrocytomas of childhood are pilocytic.

Pilocytic astrocytomas may rarely occur in adults. They are usually treated by surgical resection and in most cases have a favorable prognosis. 